Phonon interference in crystalline and amorphous confined nanoscopic films

Zhi Liang, Thomas E. Wilson, and Pawel Keblinski

Citation: J. Appl. Phys. 121, 075303 (2017); doi: 10.1063/1.4976563

View online: http://dx.doi.org/10.1063/1.4976563

View Table of Contents: http://aip.scitation.org/toc/jap/121/7

Published by the American Institute of Physics

AIP $\left.\right|_{\text {Applied Physics }} ^{\text {Journal of }}$

INTRODUCING INVITED PERSPECTIVES

Ultrafast magnetism and $\mathrm{THz}$ spintronics

Authors: Jakob Walowski and Markus Münzenberg 


\title{
Phonon interference in crystalline and amorphous confined nanoscopic films
}

\author{
Zhi Liang, ${ }^{1, a)}$ Thomas E. Wilson, ${ }^{2}$ and Pawel Keblinski ${ }^{3, b)}$ \\ ${ }_{1}^{1}$ Department of Mechanical Engineering, California State University, Fresno, Fresno, California 93740, USA \\ ${ }^{2}$ Department of Physics, Marshall University, Huntington, West Virginia 25755, USA \\ ${ }^{3}$ Department of Materials Science and Engineering, Rensselaer Polytechnic Institute, Troy, New York 12180, \\ USA
}

(Received 29 November 2016; accepted 1 February 2017; published online 17 February 2017)

\begin{abstract}
Using molecular dynamics phonon wave packet simulations, we study phonon transmission across hexagonal $(h)$ - $\mathrm{BN}$ and amorphous silica $\left(a-\mathrm{SiO}_{2}\right)$ nanoscopic thin films sandwiched by two crystalline leads. Due to the phonon interference effect, the frequency-dependent phonon transmission coefficient in the case of the crystalline film ( $\mathrm{Si}|h-\mathrm{BN}| \mathrm{Al}$ heterostructure) exhibits a strongly oscillatory behavior. In the case of the amorphous film $\left(\mathrm{Si}\left|a-\mathrm{SiO}_{2}\right| \mathrm{Al}\right.$ and $\mathrm{Si}\left|a-\mathrm{SiO}_{2}\right| \mathrm{Si}$ heterostructures), in spite of structural disorder, the phonon transmission coefficient also exhibits oscillatory behavior at low frequencies (up to $\sim 1.2 \mathrm{THz}$ ), with a period of oscillation consistent with the prediction from the two-beam interference equation. Above $1.2 \mathrm{THz}$, however, the phonon interference effect is greatly weakened by the diffuse scattering of higher-frequency phonons within an $a-\mathrm{SiO}_{2}$ thin film and at the two interfaces confining the $a-\mathrm{SiO}_{2}$ thin film. Published by AIP Publishing.

[http://dx.doi.org/10.1063/1.4976563]
\end{abstract}

\section{INTRODUCTION}

Phonons are the primary thermal energy carriers in semiconductor devices. As the size of semiconductor components in microelectronics reduces to the nanoscale, phonon scattering at material interfaces can strongly affect thermal transport in nanostructured components. It was found in numerous experiments and numerical simulations that the specular reflection and transmission of phonon waves at interfaces of nanostructured components may result in phonon interference effects which can be used for the modification of phonon dispersion and for controlling nanoscale heat transport. ${ }^{1-9}$

To achieve strong phonon interference effects, the thickness of confined thin films should be smaller than the phonon mean free path (MFP) such that the phonons can travel ballistically between two interfaces of the thin film. Phonon MFPs of typical crystalline semiconductors such as $\mathrm{Si}, \mathrm{GaN}$, and graphite are on the order of tens of nanometers to micrometers. ${ }^{10-12}$ Accordingly, evident phonon interference effects were observed by both experimental work and theoretical analysis on phonon transport across superlattices, i.e., alternative nanoscopic layers of epitaxially bonded semiconductors such as GaAs and AlAs. ${ }^{2,13-15}$

For amorphous materials such as amorphous silica ( $a$ $\mathrm{SiO}_{2}$ ), however, the traveling phonons can be diffusely scattered by the disordered structure, which significantly limits the phonon MFP. As phonons are diffusely scattered, the phases of phonons could be randomized such that the phonon interference effects become negligible. $a-\mathrm{SiO}_{2}$ is often used as a thin layer of insulating material separating single crystalline $\mathrm{Si}$ in the silicon on insulator (SOI) wafers, which are

\footnotetext{
a)Electronic mail: zliang@csufresno.edu

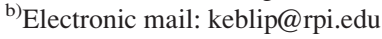

the starting point for a variety of advanced devices. ${ }^{16}$ As a dielectric material, $a-\mathrm{SiO}_{2}$ thin films are also used as a planar capacitor in the traditional metal-oxide-semiconductor (MOS) structure. ${ }^{17}$ Due to the importance of the amorphous thin film in a variety of applications, several recent studies on thermal/phonon transport across crystalline|amorphous heterojunctions were carried out. ${ }^{18-20}$ Specifically, Deng et al. ${ }^{19}$ studied phonon transport across a $\mathrm{Si}\left|a-\mathrm{SiO}_{2}\right| \mathrm{Si}$ structure using a phonon wave packet (WP) method and found that the phonon transmission coefficient decreases almost monotonically with increasing phonon frequency. No evident phonon interference effects were observed in the work of Deng et al. ${ }^{19}$ partially due to the fact that the frequency interval in their work is $\sim 1 \mathrm{THz}$, which is comparable with the expected period of oscillation.

Our recent study on phonon properties in bulk $a-\mathrm{SiO}_{2}$ shows that the sound attenuation coefficient, i.e., the inverse of MFP of acoustic phonons, roughly follows the Rayleighlike fourth power frequency dependence. ${ }^{21}$ As the MFP of phonons reduces rapidly with increasing phonon frequency, the MFP of phonons can be comparable to their wavelength. In this case, the Ioffe-Regel (IR) crossover, where the phonon mean free path is comparable to its wavelength, is reached. ${ }^{22,23}$ Our recent MD simulations show that the IR crossover frequency for both longitudinal and transverse phonons in $a-\mathrm{SiO}_{2}$ is $\sim 1.4 \mathrm{THz} .{ }^{21}$ Above the IR crossover frequency, the phonon might become nonpropagating and/or localized. ${ }^{24-26}$ Therefore, it is reasonable to expect negligible phonon interference effects for phonon frequency much greater than $1.4 \mathrm{THz}$.

Below $1.4 \mathrm{THz}$, however, the MFP of phonons in $a$ $\mathrm{SiO}_{2}$ increases very fast to values over $10 \mathrm{~nm}$ as phonon frequency reduces. ${ }^{21}$ This indicates that the low-frequency phonons have a high possibility of travelling ballistically 
between two interfaces of nanoscopic $a-\mathrm{SiO}_{2}$ layers. Furthermore, our previous WP simulations ${ }^{21}$ show that the phonon transmission coefficient at the $\mathrm{Si} \mid a-\mathrm{SiO}_{2}$ planar interface is close to that predicted by the acoustic mismatch model $^{27}$ if the phonon frequency is lower than $1 \mathrm{THz}$. This indicates that the reflection and transmission of lowfrequency phonon waves at the $\mathrm{Si} \mid a-\mathrm{SiO}_{2}$ planar interface are essentially nondiffuse, which is necessary to phonon interference. In this work, therefore, we use molecular dynamics (MD) phonon WP simulations to study phonon transport in heterostructures containing $a-\mathrm{SiO}_{2}$ thin films to investigate if evident phonon interference effects can be observed for low-frequency acoustic phonons. The modeling results will provide a reference for future experimental investigations of sound attenuation and phonon interference in $a-\mathrm{SiO}_{2}$ nanolayers which were often a part of SOI wafers and MOS structures.

\section{SIMULATION METHOD}

\section{A. The MD model}

Figure 1 shows the three model heterostructures studied in this work. The $\mathrm{Si}\left|a-\mathrm{SiO}_{2}\right| \mathrm{Si}$ and $\mathrm{Si}\left|a-\mathrm{SiO}_{2}\right| \mathrm{Al}$ structures mimic the heterostructures in SOI wafers and traditional MOS structures, respectively. Both structures contain an amorphous $\mathrm{SiO}_{2}$ layer with a thickness of $4.6 \mathrm{~nm}$ which is close to that found in some experiments. ${ }^{28}$ For comparison, we also model the $\mathrm{Si}|h-\mathrm{BN}| \mathrm{Al}$ structure, i.e., the crystalline nanoscopic layer, which contains $15 \mathrm{~h}$-BN layers whose [1120], [1 $\overline{1} 00]$, and [0001] directions are aligned, respectively, in the $\mathrm{x}, \mathrm{y}$, and $\mathrm{z}$ directions. In all three structures, the [001] direction of the $\mathrm{Si}$ lead and the [111] direction of the Al lead are aligned in the $\mathrm{z}$ direction and the length of $\mathrm{Si}$ and $\mathrm{Al}$ leads in the $\mathrm{z}$ direction is 400 and 300 unit cells, respectively. The [1 10] and $[11 \overline{2}]$ directions of $\mathrm{Al}$ are aligned in the $\mathrm{x}$ and $\mathrm{y}$ directions, respectively. Periodic boundary conditions (PBCs) are applied in the $\mathrm{x}$ and $\mathrm{y}$ directions. In the $\mathrm{z}$ direction, the simulation box is bordered by free boundaries.

The interatomic interactions within $\mathrm{Si}, \mathrm{SiO}_{2}$, and at the interface between the two materials are modeled by the Tersoff potential with the parameters developed by Munetoh et $a l .^{29,30}$ This potential has been used to study the phonon properties of $a-\mathrm{SiO}_{2}$ and thermal/phonon transport across the $\mathrm{Si} \mid a-\mathrm{SiO}_{2}$ interface. ${ }^{20,21}$ Using the Tersoff potential, our MD simulation predicts a cubic lattice constant of $5.432 \AA$ for $\mathrm{Si}$. The Al-Al interactions are modeled by the embedded-atommethod (EAM) potential, ${ }^{31}$ which predicts a FCC lattice constant of $4.015 \AA$ for Al. The Tersoff potential with the parameters developed by Verma et al. $^{32}$ and the Lennard-Jones (LJ) potential with parameters $\varepsilon=5.0 \mathrm{meV}$ and $\sigma=3.35 \AA$ (Ref. 33) are used for intralayer and interlayer interactions in h-BN, respectively. With the Tersoff and LJ potentials, the MD simulation predicts a lattice constant of $2.522 \AA$ (BN bond length of $1.456 \AA$ ) and an interlayer distance of $3.345 \AA$ in h-BN, both of which agree well with the experimental data. ${ }^{34}$

The LJ potential for interlayer interactions in h-BN is also used for the interatomic interactions at the Si|BN interface. Allowing for the strong bonding at the $\mathrm{Al} \mid \mathrm{BN}$ interface, ${ }^{35}$ the $\mathrm{LJ}$ potential with parameters $\varepsilon=110 \mathrm{meV}$ and $\sigma=3.35 \AA$ is employed for interatomic interactions at the $\mathrm{Al} \mid \mathrm{BN}$ interface. The $\mathrm{LJ}$ potential is also used for interatomic interactions at the $a-\mathrm{SiO}_{2} \mid \mathrm{Al}$ interface. Based on $\mathrm{Si}-\mathrm{Al}$ and Al-O bond lengths, ${ }^{36}$ we set $\sigma_{A l-S i}=2.25 \AA$ and $\sigma_{A l-O}$ $=1.70 \AA$. We further set $\varepsilon_{A l-S i}=\varepsilon_{A l-O}=100 \mathrm{meV}$ such that the thermal conductance at the $\mathrm{Si} \mid \mathrm{Al}$ and $\mathrm{Si}\left|a-\mathrm{SiO}_{2}\right| \mathrm{Al}$ interfaces obtained from MD simulations is consistent with the experimental data. ${ }^{37}$ The cutoff distance for all LJ interactions is $11 \AA$ in the MD simulation.

In the initial structure of $\mathrm{Si}|h-\mathrm{BN}| \mathrm{Al}$, to make the $\mathrm{Si}$, $\mathrm{BN}$, and $\mathrm{Al}$ structures commensurate in the $\mathrm{x}$ and $\mathrm{y}$ directions, we strain the cross section of $\mathrm{Si}$ and $\mathrm{Al}$ and slightly stretch BN layers. The cross section of the structure is fixed
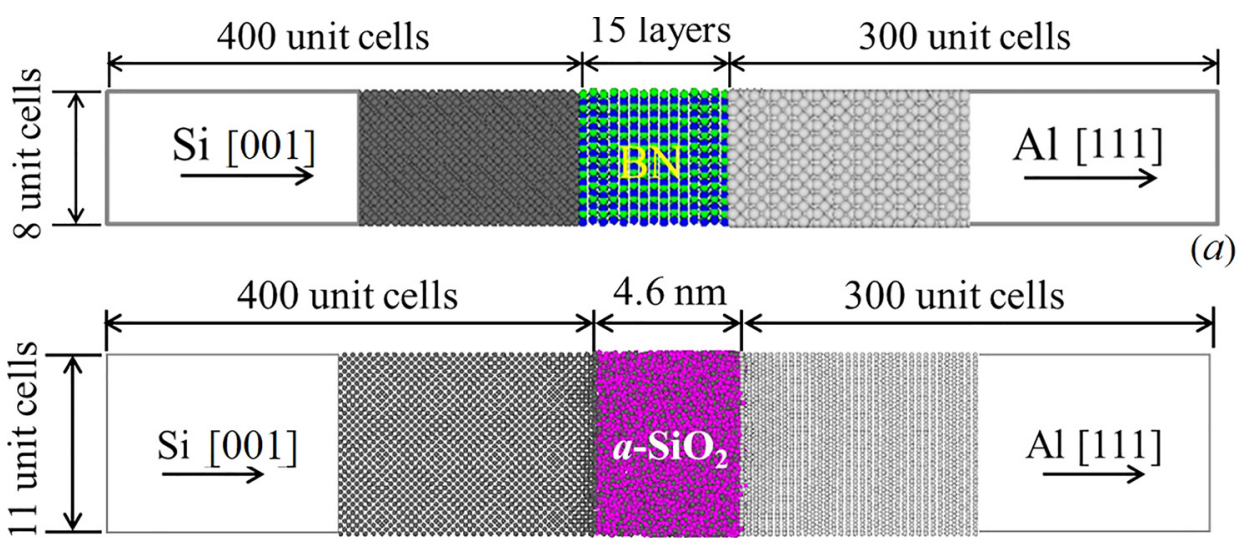

(b)

FIG. 1. Schematic diagrams of (a) the $\mathrm{Si} \mid h$-BN $\mid \mathrm{Al}$ structure, (b) the $\mathrm{Si} \mid a$ $\mathrm{SiO}_{2} \mid \mathrm{Al}$ structure, and (c) the $\mathrm{Si} \mid a$ $\mathrm{SiO}_{2} \mid \mathrm{Si}$ structure used in MD simulations. The central part of each figure shows the snapshot of atoms near the confined thin film.

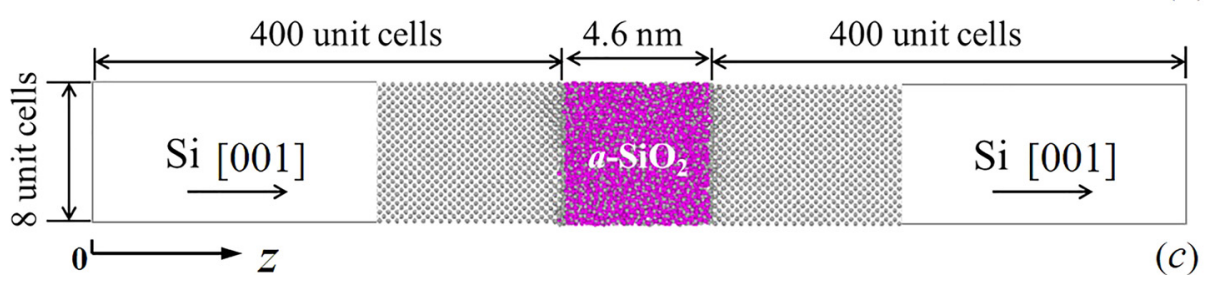


at $43.74 \AA \times 48.39 \AA$ in the simulation. In the initial structure of $\mathrm{Si}\left|a-\mathrm{SiO}_{2}\right| \mathrm{Al}$, the cross section of $\mathrm{Al}$ is slightly strained to fit the lattice constant of $\mathrm{Si}$. In the initial structure of $\mathrm{Si} \mid a$ $\mathrm{SiO}_{2} \mid \mathrm{Si}$, there is no strain in the two $\mathrm{Si}$ leads. The cross section of the $\mathrm{Si}\left|a-\mathrm{SiO}_{2}\right| \mathrm{Al}$ and $\mathrm{Si}\left|a-\mathrm{SiO}_{2}\right| \mathrm{Si}$ structure is fixed at $48.88 \AA \times 59.75 \AA$ and $43.46 \AA \times 43.46 \AA$, respectively.

The $a-\mathrm{SiO}_{2}$ thin film in the MD model is first generated by melting $\beta$-Cristobalite $\mathrm{SiO}_{2}$ in a cubic supercell with PBCs and subsequently quenching the melt to a low temperature. The simulation details are described in our previous work. ${ }^{21}$ The bulk $a-\mathrm{SiO}_{2}$ is then cut to fit the cross section of $\mathrm{Si}\left|a-\mathrm{SiO}_{2}\right| \mathrm{Si}$ and $\mathrm{Si}\left|a-\mathrm{SiO}_{2}\right| \mathrm{Al}$ structures and sandwiched by the two crystalline leads in the structure. In order to form a well-equilibrated crystalline|amorphous interface, we fix the temperature in the two crystalline leads to a temperature of $200 \mathrm{~K}$ and equilibrate the $a-\mathrm{SiO}_{2}$ to a temperature of $4000 \mathrm{~K}$ for $4 \mathrm{~ns}$. Subsequently, the $a-\mathrm{SiO}_{2}$ thin film is quenched to $200 \mathrm{~K}$ with a speed of $1 \mathrm{~K} / \mathrm{ps}$. Finally, we conduct an energy minimization of the whole structure at a temperature of $0 \mathrm{~K}$. The final equilibrated structure near the junction is shown in Fig. 1.

\section{B. The MD phonon wave-packed (WP) simulations}

Prior to each MD phonon WP simulation run, we perform an energy minimization of the structure at $0 \mathrm{~K}$ to remove the artificial mechanical stresses present in an asprepared heterogeneous system. In all simulations, a velocity Verlet algorithm with a time step size of $0.5 \mathrm{fs}$ is used for the integration of equations of motions. ${ }^{38}$

Using the equilibrated structure, we first calculate the phonon dispersion relation, including eigenvalues and eigenvectors by diagonalizing the dynamical matrix of the $\mathrm{Si}, \mathrm{Al}$, and h-BN crystals. To determine the harmonic force constants in the dynamical matrix, we displace the atoms in the center of the $\mathrm{Si}$ and $\mathrm{Al}$ leads and the h-BN thin film by $\pm 10^{-5} \AA$ from the equilibrium position in three directions. The force constants are obtained from the second order derivatives of potential energy. The phonon dispersion relation in $a-\mathrm{SiO}_{2}$ is determined by calculating the longitudinal and transverse dynamical structure factors in $a-\mathrm{SiO}_{2}$ using equilibrium MD simulations. The details of calculations can be found in our previous work. ${ }^{21}$

With the calculated eigenvalues and eigenvectors of the Si crystal, we can launch a TA/LA phonon wave packet in the center of $\mathrm{Si}$ lead at any frequency below $1.5 \mathrm{THz}$. To generate a phonon wave packet centered at a wavevector $k_{0}$ in $\lambda$ branch, and localized in space around $z_{0}$ with a spatial extent of $\sim 1 / \eta$, we displace the atoms according to ${ }^{39,40}$

$$
u_{l}^{\alpha}(s)=\frac{1}{\sqrt{M_{s}}} Q_{\lambda \vec{k}_{0}} \varepsilon_{\lambda \vec{k}_{0}}^{\alpha}(s) e^{i k_{0}\left(z_{l}-z_{0}\right)} e^{-\eta^{2}\left(z_{l}-z_{0}\right)^{2}},
$$

where $u_{l}^{\alpha}(s)$ represents the $\alpha$ component of displacement of atom $s$ in primitive cell $l$ of the Si crystal, $M_{\mathrm{S}}$ is the mass of atom $s, Q_{\lambda k 0}$ is the amplitude of the wave, $\varepsilon_{\lambda k O}{ }^{\alpha}(s)$ is the $\alpha$ component of the eigenvector of atom $s$ for $\lambda$ branch at $k_{0}$, and $z_{l}$ is the $\mathrm{z}$ coordinate of the primitive cell $l$. In the simulation, we set $Q_{\lambda k 0}=10^{-4} \AA$ and $1 / \eta=65$ unit cells. To form a wave packet that is localized in both real space and wavevector space as described in Eq. (1), the initial atomic displacements are expressed in terms of a linear combination of vibrational eigenstates ${ }^{40,41}$

$$
u_{l}^{\alpha}(s)=\frac{1}{\sqrt{N M_{s}}} \sum_{\lambda \vec{k}} Q_{\lambda \vec{k}} \varepsilon_{\lambda \vec{k}}^{\alpha}(s) e^{i k\left(z_{l}-z_{0}\right)},
$$

where $N$ is the number of primitive cells in the Si crystal. In Eq. (2), the amplitude of each vibrational normal mode, $Q_{\lambda k}$, is determined by the inverse Fourier transform of the function in Eq. (1). To determine initial atomic velocities, we add time dependence to the displacements in Eq. (2) and differentiate with respect to time. Hence, the initial velocities are given by ${ }^{40}$

$$
\dot{u}_{l}^{\alpha}(s)=\frac{1}{\sqrt{N M_{s}}} \sum_{\lambda \vec{k}}-i \omega_{\lambda \vec{k}} Q_{\lambda \vec{k}} \varepsilon_{\lambda \vec{k}}^{\alpha}(s) e^{i k\left(z_{l}-z_{0}\right)},
$$

where $\omega_{\lambda k}$ is the eigenvalue for the $\lambda$ branch at $k$. After the wave packet at a given mode and frequency is launched, we run $\mathrm{MD}$ simulation to study the propagation and scattering of the phonon wave packet in the heterostructure. Recent experimental work ${ }^{37}$ on phonon transport across $\mathrm{Si} \mid \mathrm{a}$ $\mathrm{SiO}_{2} \mid \mathrm{Al}$ interfaces indicates that direct electron-phonon coupling has negligible effects on interfacial phonon transport. Hence, the direct electron-phonon coupling at the h-BN|Al and $a-\mathrm{SiO}_{2} \mid \mathrm{Al}$ interfaces is neglected in WP simulations.

\section{SIMULATION RESULTS}

\section{A. Phonon dispersion relations.}

The calculated phonon dispersion relations for $\mathrm{Si}$ and $\mathrm{Al}$ crystals are shown in Figs. 2(a) and 2(b). The calculated results agree well with the experimental data. ${ }^{42,43}$ The split of the two dispersion curves of the TA mode in Fig. 2(b) is due to the fact that the cross section of $\mathrm{Al}$ lead is strained by $1.2 \%$ in the $\mathrm{x}$ direction and $-0.3 \%$ in the $\mathrm{y}$ direction in the $\mathrm{Si}|h-\mathrm{BN}| \mathrm{Al}$ structure. Compared to the dispersion curves from the experiment, ${ }^{44}$ Fig. 2(c) gives poor descriptions of the acoustic phonon modes in h-BN. Specifically, Fig. 2(c) underestimates the maximum frequency of the transverse mode and overestimates the maximum frequency of the longitudinal mode. The poor agreement stems mainly from the fact that the LJ parameters for interlayer interactions in $\mathrm{h}$ $\mathrm{BN}$ were fitted to match the interlayer spacing and binding energy in h-BN. ${ }^{33}$ To have a better description of the dispersion relation, a more complex interaction potential is needed. However, the purpose of this work is not to accurately predict the transmission coefficient across the $\mathrm{Si}|h-\mathrm{BN}| \mathrm{Al}$ structure. We are merely using the $\mathrm{Si}|h-\mathrm{BN}| \mathrm{Al}$ structure as a reference to show the clear phonon interference effects in the case of a confined crystalline thin film. Figure 2(d) shows phonon dispersion curves of acoustic modes in $a-\mathrm{SiO}_{2}$. We have shown in our previous work ${ }^{21}$ that the sound speeds obtained from the dispersion curve agree with the experimental data ${ }^{45,46}$ very well. 

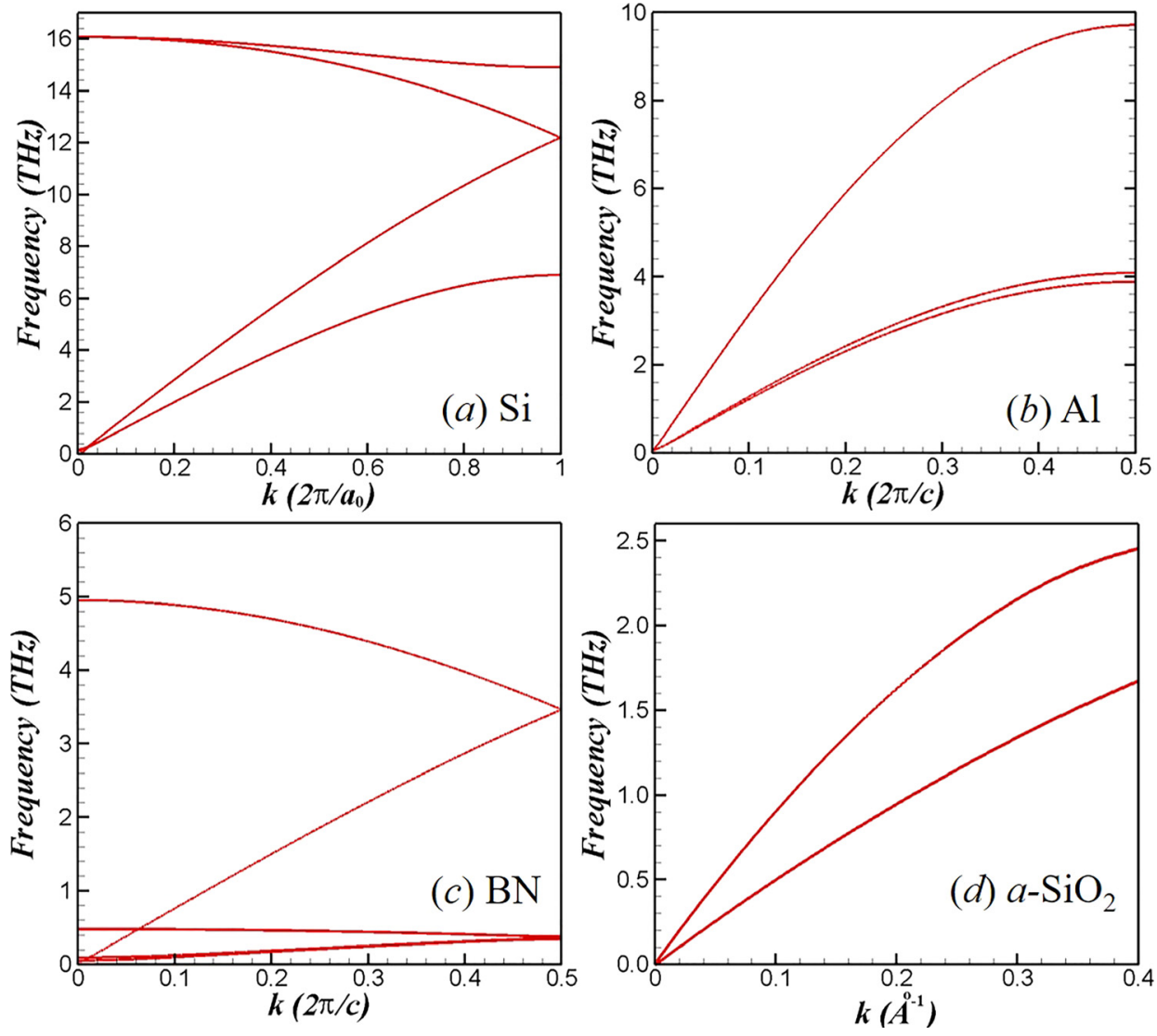

FIG. 2. The phonon dispersion curve (a) along the [001] direction of $\mathrm{Si},(\mathrm{b})$ along the [0001] direction of $h$-BN (high frequency optical modes are not shown), (c) along the [111] direction of $\mathrm{Al}$, and (d) for longitudinal and transverse phonons in $a-\mathrm{SiO}_{2}$.

\section{B. Phonon transport across the $\mathrm{Si} \mid \boldsymbol{h}$-BN $\mid \mathrm{Al}$ interface}

Figure 3(a) shows snapshots of spatial distribution of $v_{z}$ in the $\mathrm{Si} \mid h$-BN $\mid \mathrm{Al}$ structure for an LA wave packet centered at $1.5 \mathrm{THz}$. It is seen that the wave packet travels in the zdirection and the phonon transmission and reflection at the $\mathrm{Si} \mid h$-BN and $h$-BN $\mid \mathrm{Al}$ interfaces complete within 25 ps. The total energy in the $\mathrm{Si}, \mathrm{Al}$ leads, and h-BN thin film as a function of time is shown in Figs. 4(a) and 4(b). It is seen in Fig. 4(b) that no energy is trapped in the h-BN layer after phonon transmission and reflection complete. From Fig. 4(a), we calculate the phonon transmission coefficient by the fraction of phonon energy transmitted through the interface. In Fig. 5, we show the transmission coefficient for LA phonons as a function of phonon frequency. The transmission coefficient exhibits a strong oscillatory behavior as a function of frequency with a decaying envelope. The similar frequency dependency was also observed in phonon transport across confined few-layer grapheme, ${ }^{9}$ where the oscillatory behavior of transmission coefficient was shown to be the result of phonon interference arising from multiple scattering at the two interfaces.

To better understand the interference effect shown in Fig. 5(b), we evaluate the transmission coefficient as a function of frequency from an analytical one-dimensional model of masses connected by springs. ${ }^{47}$ As shown in Fig. 5(a), the model consists of a center region (mimicking h-BN layers) sandwiched between two semi-infinite leads (mimicking $\mathrm{Si}$ and $\mathrm{Al}$ crystals). In the one-dimensional chain model, masses are connected by harmonic springs where $m_{1}, m_{2}, m_{3}$ and $k_{1}$, $k_{2}, k_{3}$ are masses and spring constants in the left, center, and right region, respectively. The masses and spring constants are parameterized to reproduce the speed of sound of $\mathrm{Si}$, h$\mathrm{BN}$, and $\mathrm{Al}$ in the cross-plane direction. The two semiinfinite chains are coupled with the center part by spring constants $k_{12}$ and $k_{23}$ which are parameterized to model the $\mathrm{LJ}$ potential bonding strength at the $\mathrm{Si} \mid \mathrm{h}-\mathrm{BN}$ and $\mathrm{h}-\mathrm{BN} \mid \mathrm{Al}$ interfaces. With these settings, the transmission coefficient in the one-dimensional model is then calculated by the scattering boundary method. ${ }^{47}$ As shown in Fig. 3(b), the frequency of wave packets launched in the WP simulation has a narrow Gaussian distribution with standard deviation of $\sim 0.051$ THz. The transmission coefficient obtained from WP simulations represents an average transmission coefficient over the frequency range shown in Fig. 3(b). To directly compare the analytical results with the MD phonon WP simulations, therefore, we show the average transmission coefficient over phonons representing a wave packet used in the WP simulations. It is shown in Fig. 4(b) that the prediction from a onedimensional chain model has a very good agreement with the MD phonon WP simulation results. Both the analytical and simulation results show strongly oscillatory dependence of transmission coefficient on phonon frequency. The maxima and minima of transmission coefficient are the result of constructive and destructive interferences, respectively. ${ }^{47}$

\section{Phonon transport across the $\mathrm{Si}\left|a-\mathrm{SiO}_{2}\right| \mathrm{Al}$ interface}

In amorphous materials such as $a-\mathrm{SiO}_{2}$, phonons can be diffusely scattered by the disordered structure in the material. As a result, the nature of phonon transmission through $\mathrm{Si}\left|a-\mathrm{SiO}_{2}\right| \mathrm{Al}$ is quite different from that through $\mathrm{Si}|h-\mathrm{BN}| \mathrm{Al}$. 

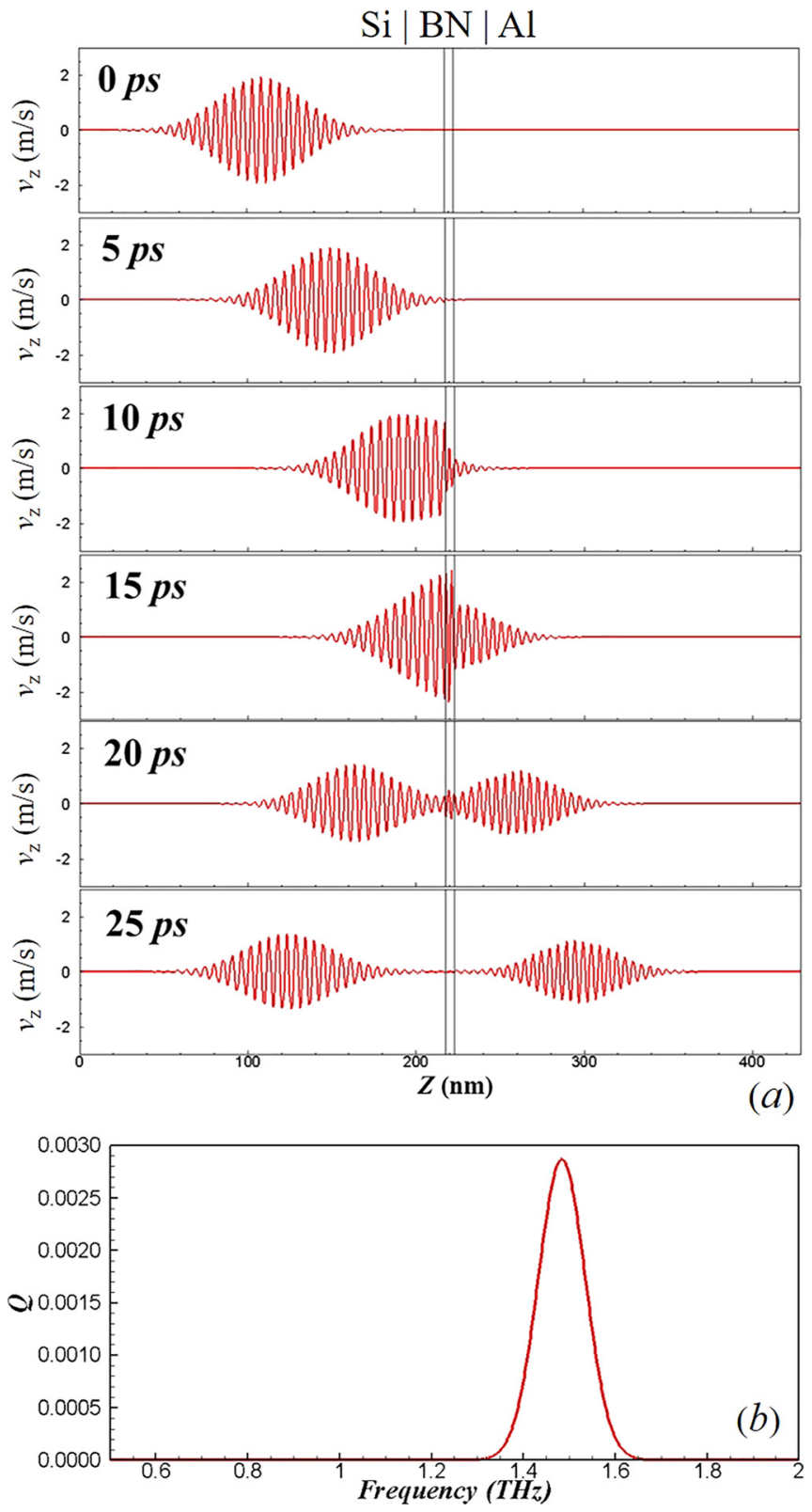

FIG. 3. (a) Snapshots of the spatial distribution of $v_{\mathrm{z}}$ in the $\mathrm{Si}|\mathrm{BN}| \mathrm{Al}$ structure for an LA mode phonon WP centered at $1.5 \mathrm{THz}$. (b) The amplitude of vibrational normal modes as a function of phonon frequency for phonon WP launched in (a).

Figures 4(c) and 4(d) show the total energy in the $\mathrm{Si}, \mathrm{Al}$ leads, and the confined $a-\mathrm{SiO}_{2}$ layer as a function of time during the WP simulation of propagation of an LA mode wave packet centered at $1.5 \mathrm{THz}$ in $\mathrm{Si}\left|a-\mathrm{SiO}_{2}\right| \mathrm{Al}$. Our previous work ${ }^{21}$ shows that the MFP of longitudinal phonons at $1.5 \mathrm{THz}$ in $a-\mathrm{SiO}_{2}$ is about $3 \mathrm{~nm}$, which is shorter than the thickness of the $a-\mathrm{SiO}_{2}$ layer $(4.6 \mathrm{~nm})$ in the $\mathrm{Si}\left|a-\mathrm{SiO}_{2}\right| \mathrm{Al}$ structure. As a result, the possibility of phonon diffuse scattering in the $a-\mathrm{SiO}_{2}$ layer is high. It is shown in Fig. 4(d) that a small portion of wave packet energy that is diffusely scattered is trapped in the $a-\mathrm{SiO}_{2}$ layer after phonon transmission completes. To calculate the phonon transmission coefficient, the transmitted phonon energy is computed as an average of total energy in the $\mathrm{Al}$ lead between 34 and 40 ps.
Below $1.5 \mathrm{THz}$, the MFP of phonons in $a-\mathrm{SiO}_{2}$ is inversely proportional to $\nu^{3.6}$ (Ref. 21$)$, where $\nu$ is the phonon frequency. For longitudinal phonons below $1 \mathrm{THz}$, the MFP increases rapidly to a value above $20 \mathrm{~nm}$ in $a-\mathrm{SiO}_{2}$, which is considerably greater than the thickness of the $a$ $\mathrm{SiO}_{2}$ layer. Moreover, the scattering low-frequency phonons at the $\mathrm{Si} \mid a-\mathrm{SiO}_{2}$ interface are essentially nondiffuse. ${ }^{21}$ Hence, we calculate the phonon transmission coefficient as a function of frequency in the range of $0.3 \mathrm{THz}$ to $1.5 \mathrm{THz}$ to explore the phonon interference effects in the $\mathrm{Si} \mid a$ $\mathrm{SiO}_{2} \mid \mathrm{Al}$ heterostructure.

Despite the disorder in the $a-\mathrm{SiO}_{2}$ layer, it is shown in Fig. 6(a) that the transmission coefficient of the LA phonon exhibits an evident oscillatory behavior as a function of frequency. To verify if the oscillatory dependence of transmission coefficient on frequency is caused by phonon interference, we compare the WP simulation results with the theoretical prediction from the two beam interference equation ${ }^{48}$

$$
I=I_{1}+I_{2}+2 \sqrt{I_{1} I_{2}} \cos \left(2 k L+\varphi_{0}\right),
$$

where $I$ is the intensity of the interference signal, i.e., the transmitted wave packet energy in our simulations; $I_{1}$ and $I_{2}$ are reflections at the two interfaces, respectively; $\varphi_{0}$ is the initial phase of the interference; $L$ is the thickness of the confined thin film, and $k$ is the wavevector of phonon waves in the thin film. Accordingly, the two adjacent interference maxima in the interference spectrum have a phase difference of $2 \pi$. Therefore,

$$
k_{p, n+1}-k_{p, n}=\frac{\pi}{L}
$$

where $k_{\mathrm{p}, \mathrm{n}+1}$ and $k_{\mathrm{p}, \mathrm{n}}$ are the center wavevectors of two adjacent peaks in the interference spectrum.

From the WP simulation results shown in Fig. 6(a), we find that the center frequencies of the first two adjacent peaks are 0.45 and $1.00 \mathrm{THz}$, respectively. Using the phonon dispersion relation shown in Fig. 2(d), we obtain the corresponding wavevectors in $a-\mathrm{SiO}_{2}$ are 0.047 and $0.112 \AA^{-1}$, respectively. According to Eq. (5), the difference between the above two wavevectors corresponds to a thickness, $L$, of $4.8 \mathrm{~nm}$, which is consistent with the thickness $(4.6 \mathrm{~nm})$ of the $a-\mathrm{SiO}_{2}$ layer in the model structure.

Similarly, we run WP simulations to investigate the transmission coefficient of TA mode phonons across the $\mathrm{Si}\left|a-\mathrm{SiO}_{2}\right| \mathrm{Al}$ structure. The MFP of TA phonons in $a-\mathrm{SiO}_{2}$ is about half of that of LA phonons at the same frequency, ${ }^{21}$ indicating a higher possibility of diffuse scattering of TA phonons in the $a-\mathrm{SiO}_{2}$ layer. As a result, the interference effects are significantly weaker. It is shown in Fig. 6(b) that the oscillatory dependence of the TA mode transmission coefficient on phonon frequency is not so evident as that of the LA phonons. In Table I, we summarize the phonon frequencies at the first three peaks shown in Figs. 6(a) and 6(b). With these peak phonon frequencies, it is shown in Table I that the thicknesses of the $a-\mathrm{SiO}_{2}$ layer calculated by Eq. (5) are all around $4.6 \mathrm{~nm}$. This indicates that the oscillatory dependence on phonon frequency is caused by phonon interference effects. 

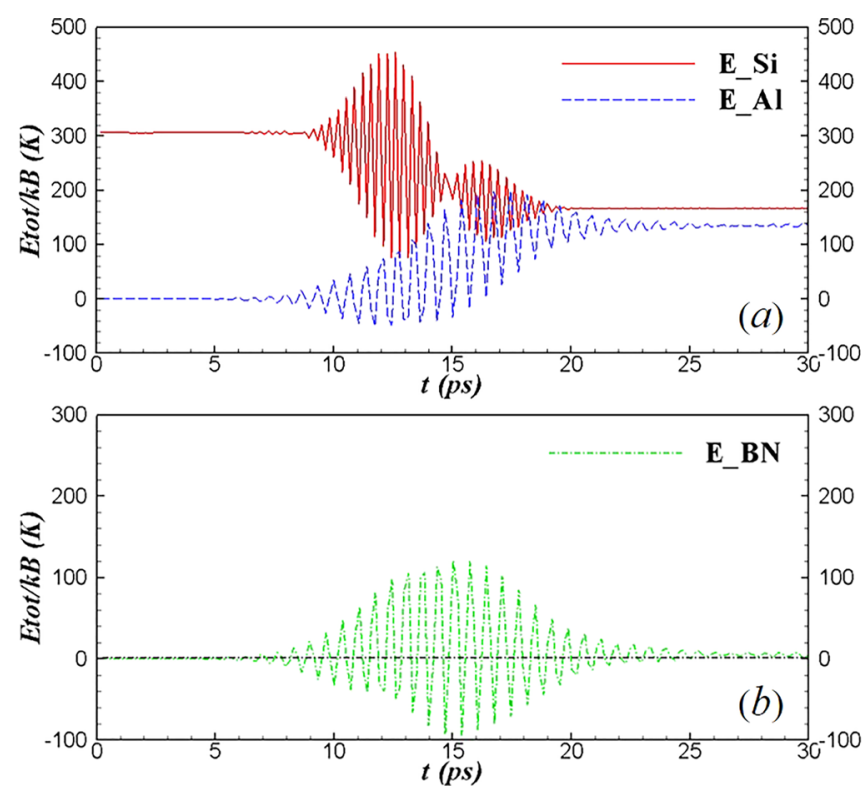
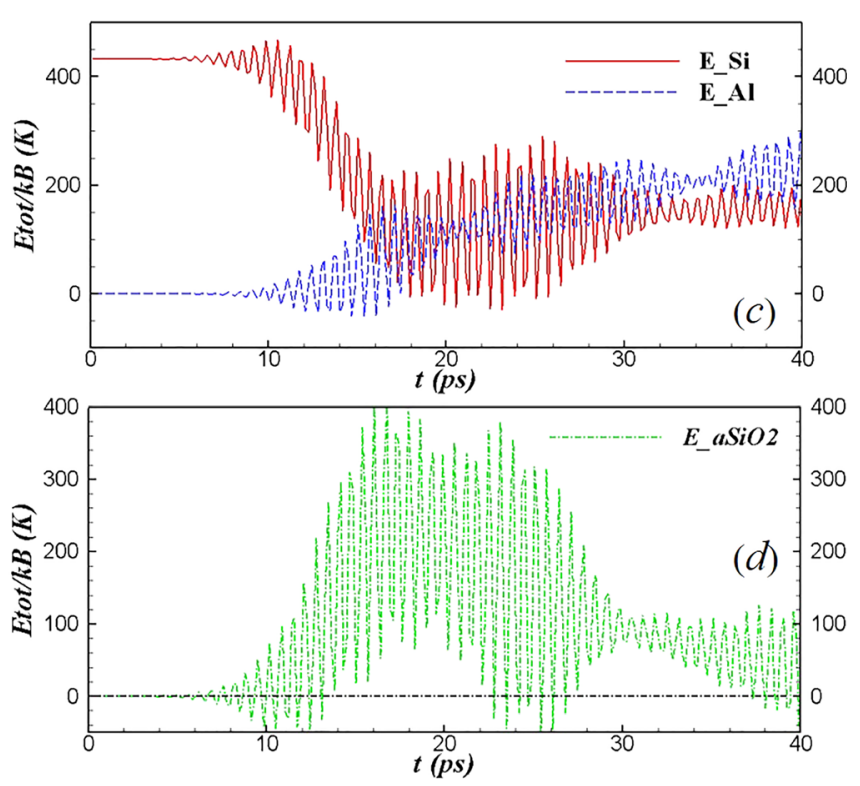

FIG. 4. The total energy as a function of time in (a) the $\mathrm{Si}$ and $\mathrm{Al}$ leads, (b) the confined $\mathrm{BN}$ thin film in the $\mathrm{Si}|\mathrm{BN}| \mathrm{Al}$ structure, (c) the $\mathrm{Si}$ and $\mathrm{Al}$ leads, and (d) the confined $a-\mathrm{SiO}_{2}$ thin film in the $\mathrm{Si}\left|a-\mathrm{SiO}_{2}\right| \mathrm{Al}$ structure during the WP simulations with a LA wave packet centered at $1.5 \mathrm{THz}$.

\section{Phonon transport across the $\mathrm{Si}\left|a-\mathrm{SiO}_{2}\right| \mathrm{Si}$ interface}

As shown in Fig. 7, the oscillatory behavior of the transmission coefficient is also found in the WP simulation of phonon transport across the $\mathrm{Si}\left|a-\mathrm{SiO}_{2}\right| \mathrm{Si}$ structure. Using a method similar to that described in Sec. III C, we first find the frequencies at peaks of the transmission coefficient in Figs. 7(a) and 7(b) and then calculate the corresponding thickness of the $a-\mathrm{SiO}_{2}$ layer by Eq. (5). It is shown in Table I that the calculated thickness agrees well with that of the $a-\mathrm{SiO}_{2}$ layer in the $\mathrm{Si}\left|a-\mathrm{SiO}_{2}\right| \mathrm{Si}$ structure. According to Eq. (5), the interval between wavevectors or frequencies of two adjacent peaks will decrease as the thickness of the $a-\mathrm{SiO}_{2}$ layer increases. To see this effect, we double the thickness of the $a-\mathrm{SiO}_{2}$ layer in the $\mathrm{Si}\left|a-\mathrm{SiO}_{2}\right| \mathrm{Si}$ structure and use WP simulation to
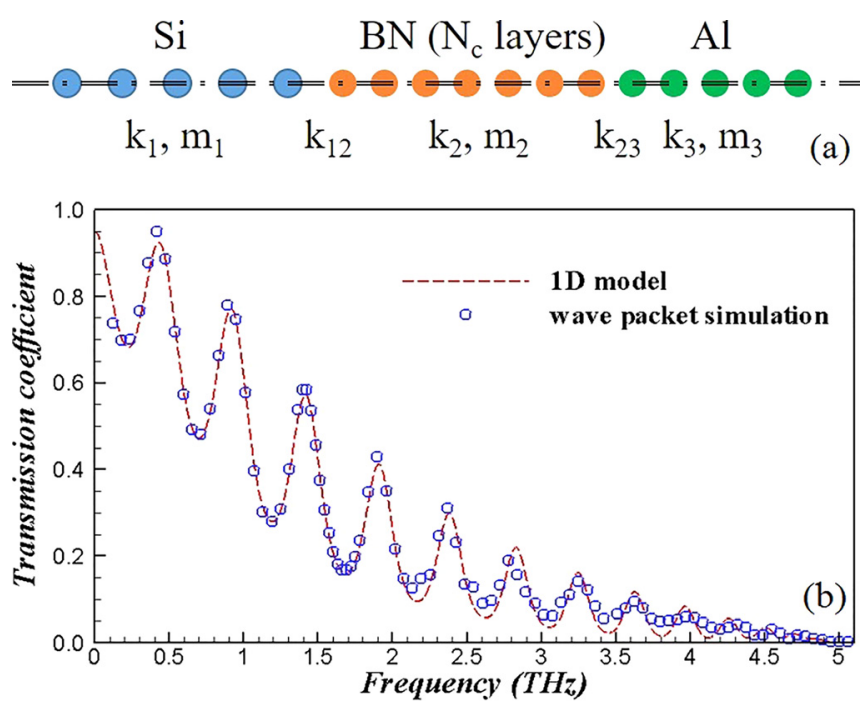

FIG. 5. (a) The schematic of the one-dimensional chain model. (b) The transmission coefficient of LA phonons in $\mathrm{Si}|\mathrm{BN}| \mathrm{Al}$ structure as a function of frequency from WP simulations and predictions from a one-dimensional chain model. calculate the dependence of the transmission coefficient on frequency. Figs. 7(c) and 7(d) show that the frequency intervals almost reduce by half as the thickness doubles. As shown in Table I, the corresponding thickness of the $a-\mathrm{SiO}_{2}$ layer calculated by Eq. (5) is consistent with the thicker $a-\mathrm{SiO}_{2}$ layer in the model structure. These results further verify that
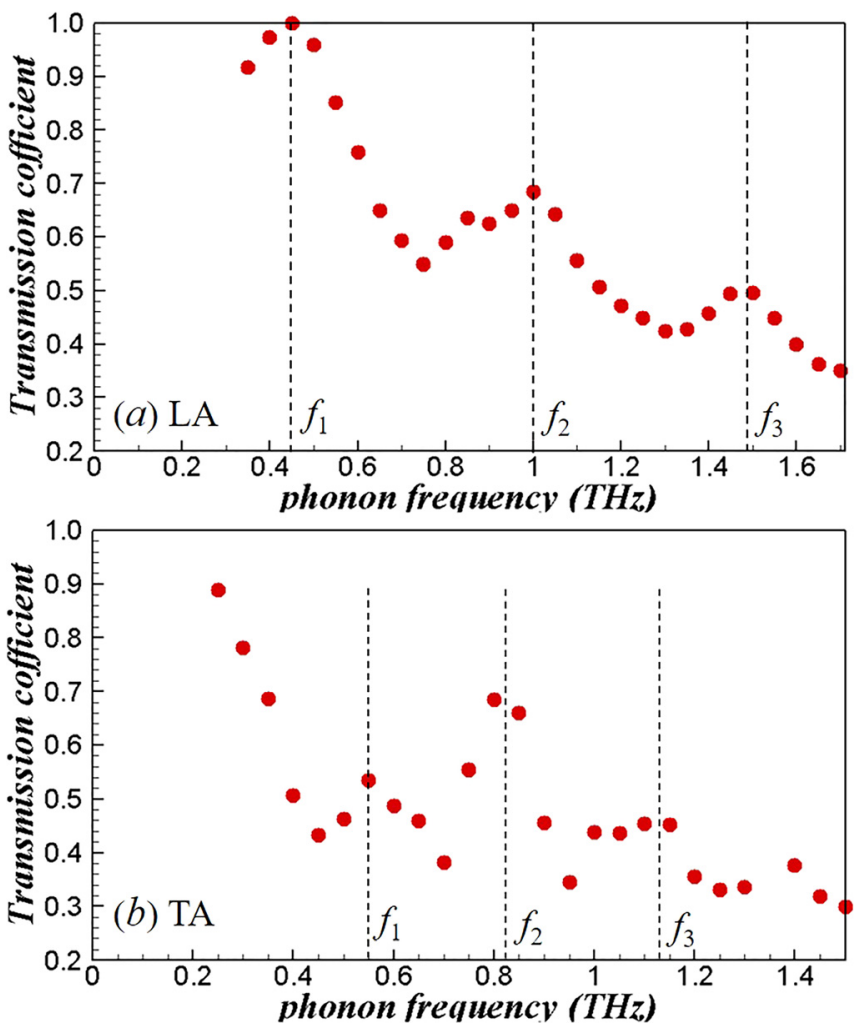

FIG. 6. The transmission coefficient for (a) LA and (b) TA phonons in a $\mathrm{Si}\left|a-\mathrm{SiO}_{2}\right| \mathrm{Al}$ structure as a function of frequency from WP simulations. The dashed lines indicate the peaks of the oscillatory transmission coefficients. 
TABLE I. Center frequencies, $\mathrm{f}_{\mathrm{p}, \mathrm{n}}$ and center wavevectors, $\mathrm{k}_{\mathrm{p}, \mathrm{n}}$ in Figs. 6 and 7. $k_{p, n+1}-k_{p, n}$ is the difference between center wavevectors of two adjacent peaks. $\mathrm{L}_{\mathrm{th}}$ is thickness of the $\mathrm{a}-\mathrm{SiO}_{2}$ layer calculated using Eq. (5).

\begin{tabular}{lccccc}
\hline \hline Model structure & $\begin{array}{c}\text { Phonon } \\
\text { mode }\end{array}$ & $\begin{array}{c}f_{\mathrm{p}, \mathrm{n}} \\
(\mathrm{THz})\end{array}$ & $\begin{array}{c}k_{\mathrm{p}, \mathrm{n}} \\
\left(\AA^{-1}\right)\end{array}$ & $\begin{array}{c}k_{\mathrm{p}, \mathrm{n}+1}-k_{\mathrm{p}, \mathrm{n}} \\
\left(\AA^{-1}\right)\end{array}$ & $\begin{array}{c}L_{\mathrm{th}} \\
(\mathrm{nm})\end{array}$ \\
\hline $\mathrm{Si}\left|a-\mathrm{SiO}_{2}\right| \mathrm{Al} L_{\mathrm{SiO} 2}=4.6 \mathrm{~nm}$ & $\mathrm{LA}$ & 0.45 & 0.047 & 0.065 & 4.8 \\
& & 1.00 & 0.112 & 0.066 & 4.8 \\
& & 1.48 & 0.178 & - & - \\
& $\mathrm{TA}$ & 0.55 & 0.111 & 0.061 & 5.2 \\
& & 0.82 & 0.172 & 0.074 & 4.2 \\
& & 1.13 & 0.246 & - & - \\
$\mathrm{Si}\left|a-\mathrm{SiO}_{2}\right| \mathrm{Si} L_{\mathrm{SiO} 2}=4.6 \mathrm{~nm}$ & $\mathrm{TA}$ & 0.42 & 0.074 & 0.072 & 4.4 \\
& & 0.71 & 0.146 & 0.067 & 4.7 \\
& & 1.00 & 0.213 & - & - \\
& LA & 0.68 & 0.074 & 0.063 & 5.0 \\
$\mathrm{Si}\left|a-\mathrm{SiO}_{2}\right| \mathrm{Si} L_{\mathrm{SiO} 2}=9.2 \mathrm{~nm}$ & & 1.19 & 0.137 & - & - \\
& & 0.40 & 0.080 & 0.035 & 9.0 \\
& & 0.57 & 0.115 & 0.040 & 7.9 \\
& & 0.75 & 0.155 & - & - \\
& LA & 0.40 & 0.041 & 0.030 & 10.5 \\
& & 0.66 & 0.071 & 0.030 & 10.5 \\
& & 0.91 & 0.101 & - & - \\
\hline \hline
\end{tabular}

the oscillatory dependence of the transmission coefficient on phonon frequency is due to phonon interference effects.

It is also seen in Fig. 7 that the phonon transmission coefficient decreases almost monotonically with increasing frequency if the phonon frequency is above $1.2 \mathrm{THz}$. Above 1.2 $\mathrm{THz}$, the MFP of phonons in $a-\mathrm{SiO}_{2}$ is comparable to or even smaller than the thickness of the $a-\mathrm{SiO}_{2}$ layer. Furthermore, the phonon scattering at $\mathrm{Si} \mid a-\mathrm{SiO}_{2}$ interfaces becomes diffuse as the phonon frequency is above 1.2 $\mathrm{THz}^{21}$ The diffuse scattering of phonons in the thin film and at the two interfaces makes phonon interference effects negligible. This is consistent with the results found in the work of Deng et al. ${ }^{19}$

\section{SUMMARY}

We carry out MD phonon WP simulations to investigate phonon transport across crystalline and amorphous nanolayers sandwiched by two crystalline leads. For phonon transport across the $\mathrm{Si} \mid h$-BN $\mid \mathrm{Al}$ structure, both WP simulations and the one-dimensional chain model show a strongly oscillatory dependence of the transmission coefficient on phonon frequency indicative of significant phonon interference effects. For phonon transport across the $\mathrm{Si}\left|a-\mathrm{SiO}_{2}\right| \mathrm{Al}$ and $\mathrm{Si}\left|a-\mathrm{SiO}_{2}\right| \mathrm{Si}$ structures, despite the disorder in $a-\mathrm{SiO}_{2}$, one can still see the oscillatory behavior of the transmission coefficient in the range of low frequencies $(<1.2 \mathrm{THz})$. The consistency between the WP simulation results and the prediction from the two-beam interference equation verifies that the oscillatory dependence on frequency is due to the phonon interference effect. The interference effect is shown to result from that fact that the MFP of low-frequency phonons in $a$ $\mathrm{SiO}_{2}$ is much longer than the thickness of $a-\mathrm{SiO}_{2}$ in the model structure and the phonon reflection and transmission at the crystalline|amporhous planar interfaces are essentially
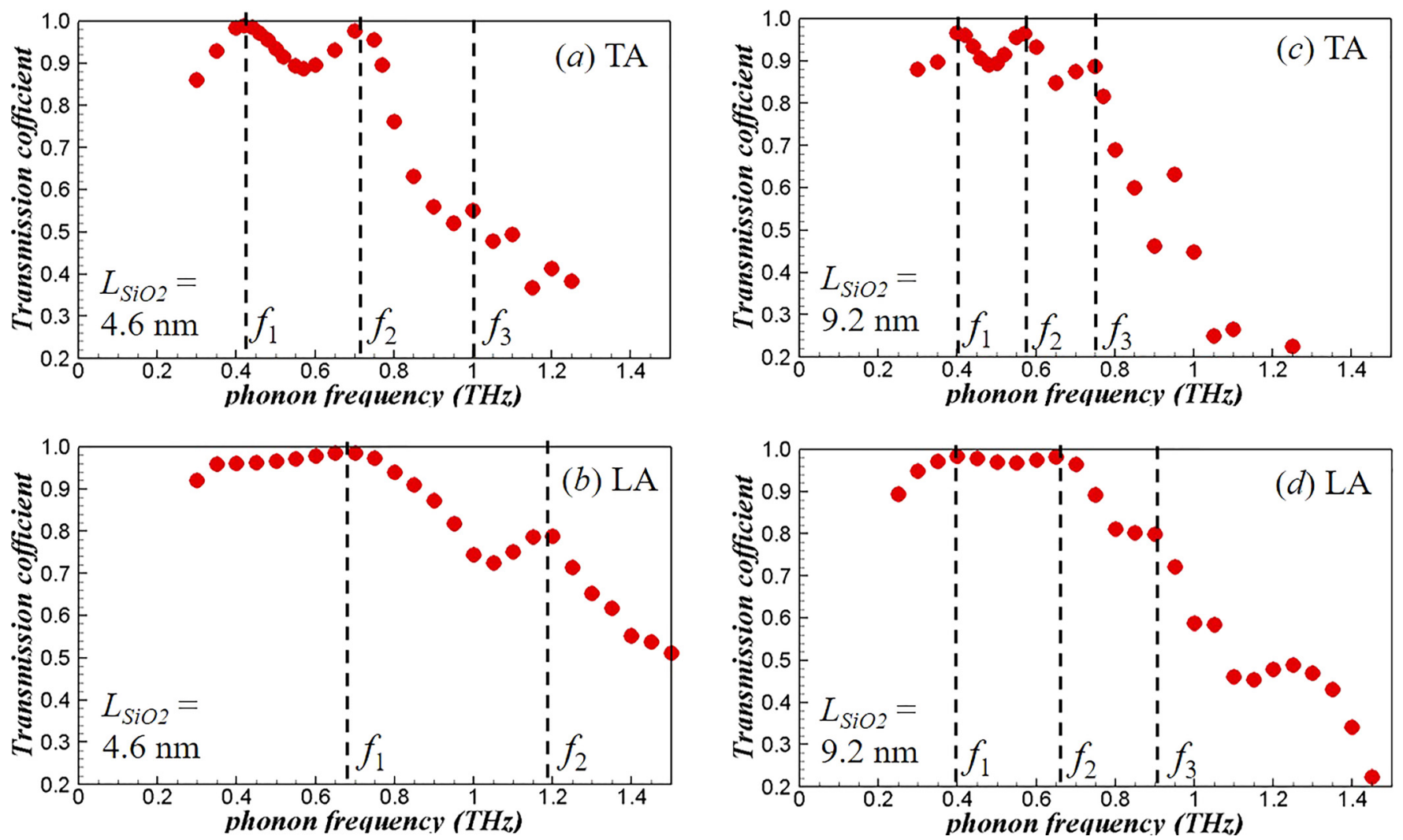

FIG. 7. The transmission coefficient for (a) LA and (b) TA phonons in a $\mathrm{Si}\left|a-\mathrm{SiO}_{2}\right| \mathrm{Si}$ structure $\left(L_{\mathrm{SiO} 2}=4.6 \mathrm{~nm}\right)$, and (c) $\mathrm{LA}$ and (d) TA phonons in the $\mathrm{Si} \mid a$ $\mathrm{SiO}_{2} \mid \mathrm{Si}$ structure $\left(L_{\mathrm{SiO} 2}=9.2 \mathrm{~nm}\right)$ as a function of frequency from WP simulations. The dashed lines indicate the peaks of the oscillatory transmission coefficients. 
nondiffuse in the range of low frequencies. In the range of high frequencies, however, the MFP of phonons in $a-\mathrm{SiO}_{2}$ reduces to a few nanometers and the interfacial scattering becomes diffuse. In this case, the diffuse phonon scattering randomizes the phases of phonons which makes the phonon interference effects negligible.

The WP simulations in this work are all carried out at zero temperature. The frequency of phonons studied in this work is near and below the IR crossover frequency of $a$ $\mathrm{SiO}_{2} \cdot{ }^{21}$ In this case, the attenuation of phonons in $a-\mathrm{SiO}_{2}$ is mainly induced by structural disorder, which is a temperature independent process. ${ }^{22}$ Therefore, the MFP of these phonons is essentially independent of temperature. In the case of finite temperature, however, the phonon scattering at the two interfaces confining the thin film might be more inelastic due to increasing anharmonicity of the atomic interactions. This could weaken the phonon interference effects.

\section{ACKNOWLEDGMENTS}

This work was supported by NASA EPSCoR project No. NNX11AM04A and by the NY State NYSTAR funded Focus Interconnect Center. We would like to thank eXtreme Science and Engineering Discovery Environment (XSEDE) for providing us supercomputer resources for MD simulations.

${ }^{1}$ M. Maldovan, Nat. Mater. 14, 667 (2015).

${ }^{2}$ M. N. Luckyanova, J. Garg, K. Esfarjani, A. Jandl, M. T. Bulsara, A. J. Schmidt, A. J. Minnich, S. Chen, M. S. Dresselhaus, Z. Ren, E. A. Fitzgerald, and G. Chen, Science 338, 936 (2012).

${ }^{3}$ J. Ravichandran, A. K. Yadav, R. Cheaito, P. B. Rossen, A. Soukiassian, S. J. Suresha, J. C. Duda, B. M. Foley, C.-H. Lee, Y. Zhu, A. W. Lichtenberger, J. E. Moore, D. A. Muller, D. G. Schlom, P. E. Hopkins, A. Majumdar, R. Ramesh, and M. A. Zurbuchen, Nat. Mater. 13, 168 (2014).

${ }^{4}$ M. V. Simkin and G. D. Mahan, Phys. Rev. Lett. 84, 927 (2000).

${ }^{5}$ N. Zen, T. A. Puurtinen, T. J. Isotalo, S. Chaudhuri, and I. J. Maasilta, Nat. Commun. 5, 3435 (2014).

${ }^{6}$ M. Maldovan, Phys. Rev. Lett. 110, 025902 (2013).

${ }^{7}$ L. Hu, L. Zhang, M. Hu, J.-S. Wang, B. Li, and P. Keblinski, Phys. Rev. B 81, 235427 (2010).

${ }^{8}$ M. Shen and P. Keblinski, J. Appl. Phys. 115, 144310 (2014).

${ }^{9}$ M. Shen, P. K. Schelling, and P. Keblinski, Phys. Rev. B 88, 045444 (2013).

${ }^{10}$ K. Esfarjani and G. Chen, Phys. Rev. B 84, 085204 (2011).

${ }^{11}$ B. A. Danilchenko, T. Paszkiewicz, S. Wolski, A. Jeżowski, and T. Plackowski, Appl. Phys. Lett. 89, 061901 (2006).

${ }^{12}$ H. Zhang, X. Chen, Y.-D. Jho, and A. J. Minnich, Nano Lett. 16, 1643 (2016).

${ }^{13}$ M. Trigo, A. Bruchhausen, A. Fainstein, B. Jusserand, and V. ThierryMieg, Phys. Rev. Lett. 89, 227402 (2002).

${ }^{14}$ Y. Ezzahri, S. Grauby, J. M. Rampnoux, H. Michel, G. Pernot, W. Claeys, S. Dilhaire, C. Rossignol, G. Zeng, and A. Shakouri, Phys. Rev. B 75, 195309 (2007).

${ }^{15}$ N. D. Lanzillotti-Kimura, A. Fainstein, B. Jusserand, A. Lemaitre, O. Mauguin, and L. Largeau, Phys. Rev. B 76, 174301 (2007).
${ }^{16}$ B.-Y. Nguyen, G. Celler, and C. Mazure, J. Integr. Circuit Syst. 4, 51 (2009), available at http://www.sbmicro.org.br/jics/html/artigos/vol4no2/ 01.pdf.

${ }^{17}$ F. Jolly, F. Rochet, G. Dufour, C. Grupp, and A. Taleb-Ibrahimi, J. NonCryst. Solids 280, 150 (2001).

${ }^{18}$ G. Ashutosh, J. L. Braun, and P. E. Hopkins, J. Appl. Phys. 119, 235305 (2016).

${ }^{19}$ B. Deng, A. Chernatynskiy, M. Khafizov, D. H. Hurley, and S. R. Phillpot, J. Appl. Phys. 115, 084910 (2014).

${ }^{20}$ E. Lampin, Q.-H. Nguyen, P. A. Francioso, and F. Cleri, Appl. Phys. Lett. 100, 131906 (2012).

${ }^{21}$ Z. Liang and P. Keblinski, Phys. Rev. B 93, 054205 (2016).

${ }^{22}$ G. Baldi, V. M. Giordano, G. Monaco, and B. Ruta, Phys. Rev. Lett. 104, 195501 (2010).

${ }^{23}$ A. F. Ioffe and A. R. Regel, Progress in Semiconductors, edited by A. F. Gibson, F. A. Kroger, and R. E. Burgess (Heywood, London, 1960), Vol. 4, p. 237.

${ }^{24}$ S. John, H. Sompolinsky, and M. J. Stephen, Phys. Rev. B 27, 5592 (1983).

${ }^{25}$ P. B. Allen, J. L. Feldman, J. Fabian, and F. Wooten, Phil. Mag. B 79, 1715 (1999).

${ }^{26}$ M. Foret, E. Courtens, R. Vacher, and J.-B. Suck, Phys. Rev. Lett. 77, 3831 (1996).

${ }^{27}$ E. T. Swartz and R. O. Pohl, Rev. Mod. Phys. 61, 605 (1989).

${ }^{28}$ D. H. Hurley, M. Khafizov, and S. L. Shinde, J. Appl. Phys. 109, 083504 (2011).

${ }^{29}$ S. Munetoh, T. Motooka, K. Moriguchi, and A. Shintani, Comput. Mater. Sci. 39, 334 (2007).

${ }^{30}$ J. Tersoff, Phys. Rev. B 38, 9902 (1988).

${ }^{31}$ K. W. Jacobsen, J. K. Norskov, and M. J. Puska, Phys. Rev. B 35, 7423 (1987).

${ }^{32}$ V. Verma, V. K. Jindal, and K. Dharamvir, Nanotechnology 18, 435711 (2007).

${ }^{33}$ I. Nikiforov, D.-M. Tang, X. Wei, T. Dumitrică, and D. Golberg, Phys. Rev. Lett. 109, 025504 (2012).

${ }^{34}$ S. L. Rumyantsev, M. E. Levinshtein, A. D. Jackson, S. N. Mohammmad, G. L. Harris, M. G. Spencer, and M. S. Shur, Properties of Advanced Semiconductor Materials GaN, AlN, InN, BN, SiC, SiGe, edited by M. E. Levinshtein, S. L. Rumyantsev, and M. S. Shur (John Wiley \& Sons, Inc., New York, 2001).

${ }^{35}$ A. V. Krasheninnikov, N. Berseneva, D. G. Kvashnin, J. Enkovaara, T. Bjorkman, P. Sorokin, D. Shtansky, R. M. Nieminen, and D. Golberg, J. Phys. Chem. C 118, 26894 (2014).

${ }^{36}$ M. O'Keefe and N. E. Brese, J. Am. Chem. Soc. 113, 3226 (1991).

${ }^{37}$ C. Hua, X. Chen, N. K. Ravichandran, and A. J. Minnich, e-print arXiv: 1509.07806 .

${ }^{38}$ D. Frenkel and B. Smit, Understanding Molecular Simulation (Academic Press, San Diego, 2002).

${ }^{39}$ P. K. Schelling, S. R. Phillpot, and P. Keblinski, App. Phys. Lett. 80, 2484 (2002).

${ }^{40}$ Z. Liang and P. Keblinski, Phys. Rev. B 90, 075411 (2014).

${ }^{41}$ P. K. Schelling, S. R. Phillpot, and P. Keblinski, J. Appl. Phys. 95, 6082 (2004).

${ }^{42}$ G. Dolling, Inelastic Scattering Neutrons in Solids and Liquids (International Atomic Energy Agency, Unesco, 1963), p. 37.

${ }^{43}$ R. Stedman and G. Nilsson, Phys. Rev. 145, 492 (1966).

${ }^{44}$ V. N. Popov, Phys. Rev. B 67, 085408 (2003).

${ }^{45}$ P. Benassi, M. Krisch, C. Masciovecchio, V. Mazzacurati, G. Monaco, G. Ruocco, F. Sette, and R. Verbeni, Phys. Rev. Lett. 77, 3835 (1996).

${ }^{46}$ F. Terki, C. Levelut, M. Boissier, and J. Pelous, Phys. Rev. B 53, 2411 (1996).

${ }^{47}$ L. F. Zhang, P. Keblinski, J. S. Wang, and B. W. Li, Phys. Rev. B 83, 064303 (2011).

${ }^{48}$ B. Qi, G. R. Pickrell, J. Xu, P. Zhang, Y. Duan, W. Peng, Z. Huang, W. Huo, H. Xiao, R. G. May, and A. Wang, Opt. Eng. 42, 3165 (2003). 